\title{
Supplementary file 01
}

McPhee, P.J., van Hinsbergen, D.J.J., and Thomson., S.

Figure S1 and S2 show age vs. effective uranium and age vs. grain size plots for AHe and ZHe results respectively.

Table S1 to S8 contain individual grain ages and compositions from AFT analysis. In addition, Table S2 contains fission track length data.

Table S9 contains vitrinite reflectance data from Hokerek et al., (2014), used in inverse thermal modelling of sample KOP-6.

Figures S3 to S8 show results of inverse thermal modelling shown in Figure 4 of the main article, plus pre-100 Ma time-temperature paths. Constraint boxes are shown in thin blue lines; good-fitting paths are pink; acceptable-fitting paths are green; weighted mean path is navy-blue. Path generation criteria are shown in white boxes. Tables in each figure show model ages and measured ages for best fitting model paths (thick black lines). 
Figure S1:

Plots of AHe age vs. effective uranium (eU)

and $\mathbf{A H e}$ age vs. grain size (equivalent spherical radius $R s$ )
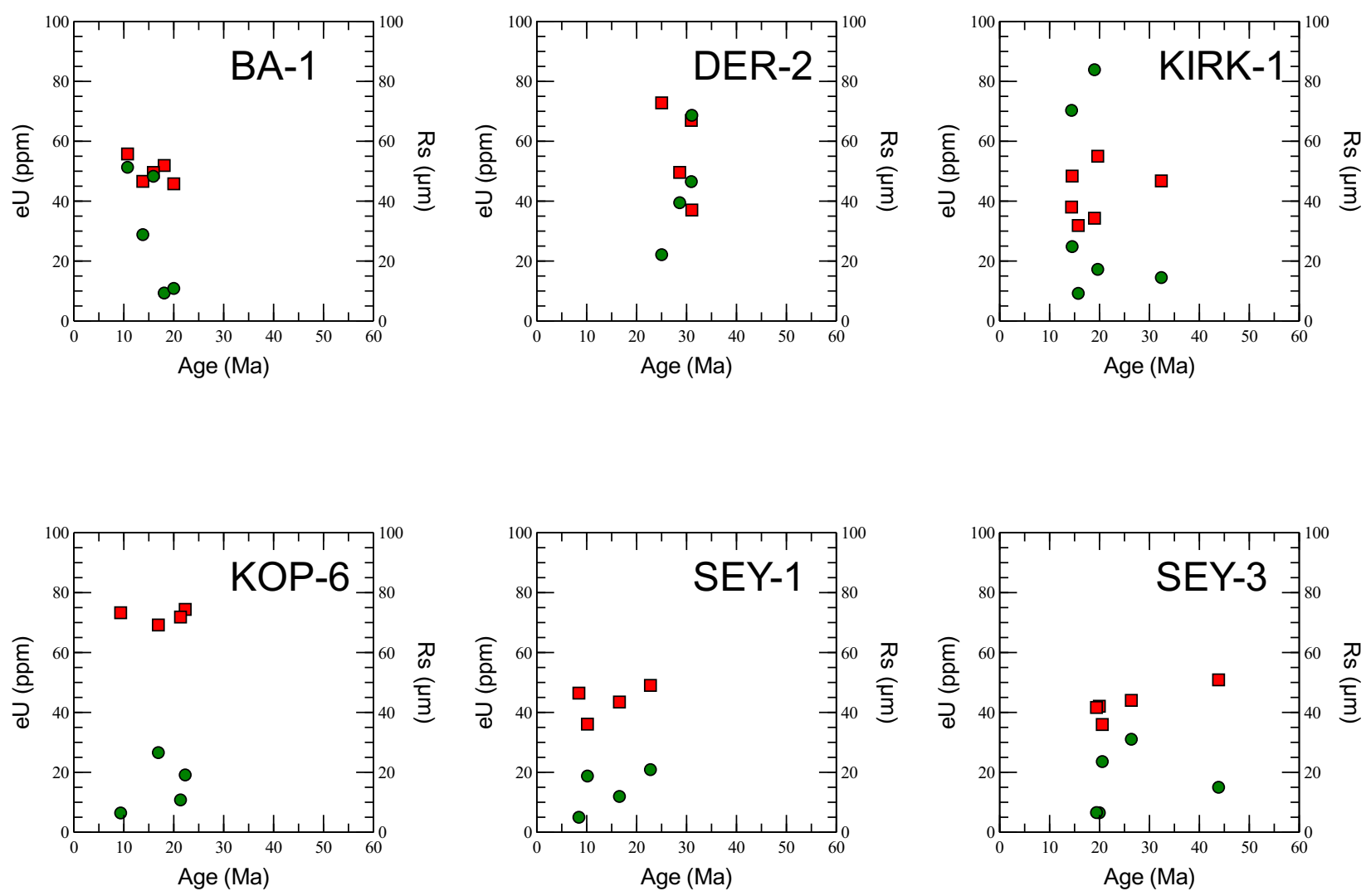

$$
\begin{array}{ll}
- & \mathrm{eU}(\mathrm{ppm}) \\
& \mathrm{Rs}(\mu \mathrm{m})
\end{array}
$$

Figure S2:

Plots of ZHe age vs. effective uranium (eU)

and $\mathbf{Z H e}$ age vs. grain size (equivalent spherical radius $R s$ )
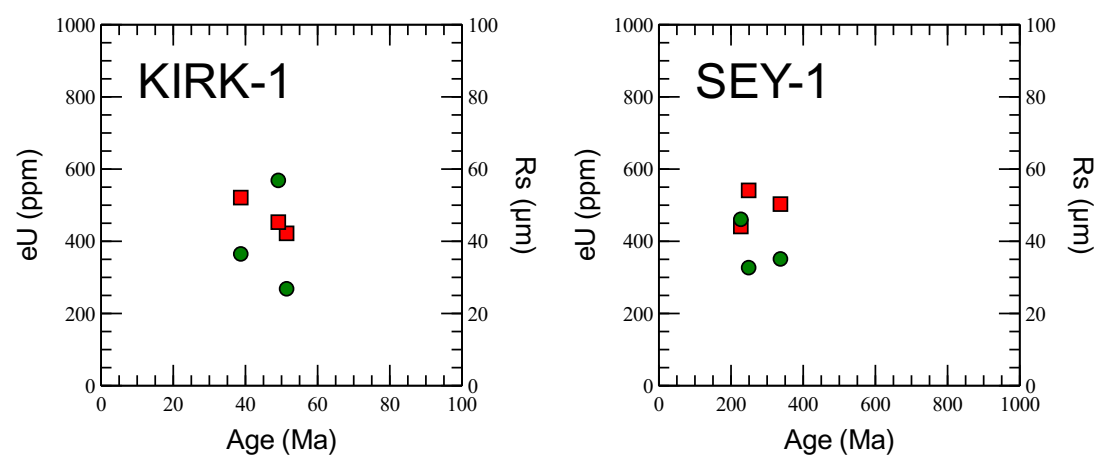
Figure S3:

Radial plots of AFT data

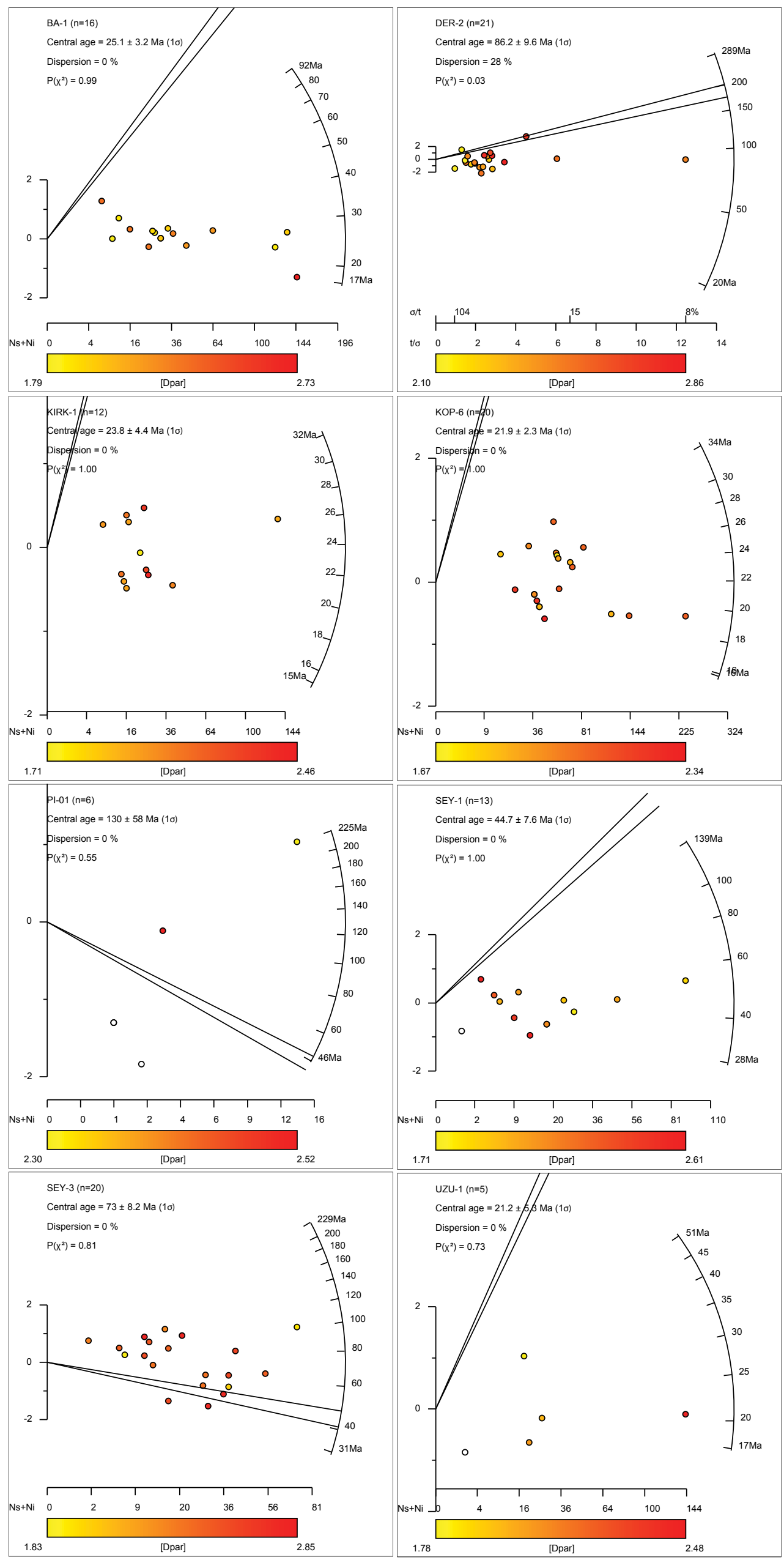


Table S1

$$
\begin{array}{lll}
\text { Sample Number } & \text { BA-1 } & \\
\text { Position (\#) } & 5 & \\
\text { Area of Graticule Square } & 6.400 \mathrm{E}-07 & \\
\text { No. of Crystals } & 16 & \\
\text { Zeta Factor } \pm \text { Error } & 368.1 & 14.9 \\
\text { Rho d (\% Relative Error) } & 1.254 \mathrm{E}+06 & 1.58 \\
\mathrm{~N} \mathrm{~d} & 4013 &
\end{array}
$$

\begin{tabular}{|c|c|c|c|c|c|c|c|c|c|c|}
\hline $\mathrm{Ns}$ & $\mathrm{Ni}$ & $\mathrm{Ng}$ & Dpar & Dper & $\rho s$ & $\rho \mathrm{i}$ & $\rho s / \rho i$ & U ppm & Age (Ma) & Age error \\
\hline 7 & 57 & 70 & 2.18 & 0.60 & $1.563 \mathrm{E}+05$ & $1.272 \mathrm{E}+06$ & 0.1228 & 15.2 & 28.28 & 11.39 \\
\hline 2 & 10 & 32 & 1.87 & 0.65 & $9.766 \mathrm{E}+04$ & $4.883 E+05$ & 0.2000 & 5.8 & 46.00 & 35.68 \\
\hline 4 & 30 & 20 & 1.98 & 0.61 & $3.125 \mathrm{E}+05$ & $2.344 \mathrm{E}+06$ & 0.1333 & 28.0 & 30.70 & 16.40 \\
\hline 4 & 41 & 60 & 2.18 & 0.57 & $1.042 E+05$ & $1.068 \mathrm{E}+06$ & 0.0976 & 12.8 & 22.48 & 11.81 \\
\hline 11 & 110 & 60 & 1.86 & 0.48 & $2.865 \mathrm{E}+05$ & $2.865 \mathrm{E}+06$ & 0.1000 & 34.3 & 23.04 & 7.35 \\
\hline 3 & 24 & 25 & 1.86 & 0.58 & $1.875 \mathrm{E}+05$ & $1.500 E+06$ & 0.1250 & 17.9 & 28.79 & 17.67 \\
\hline 4 & 33 & 18 & 2.27 & 0.45 & $3.472 E+05$ & $2.865 \mathrm{E}+06$ & 0.1212 & 34.3 & 27.92 & 14.83 \\
\hline 3 & 27 & 21 & 2.05 & 0.58 & $2.232 \mathrm{E}+05$ & $2.009 \mathrm{E}+06$ & 0.1111 & 24.0 & 25.59 & 15.62 \\
\hline 2 & 22 & 25 & 2.31 & 0.51 & $1.250 \mathrm{E}+05$ & $1.375 \mathrm{E}+06$ & 0.0909 & 16.4 & 20.95 & 15.50 \\
\hline 3 & 23 & 25 & 1.94 & 0.54 & $1.875 \mathrm{E}+05$ & $1.438 \mathrm{E}+06$ & 0.1304 & 17.2 & 30.03 & 18.48 \\
\hline 3 & 27 & 20 & 2.02 & 0.53 & $2.344 \mathrm{E}+05$ & $2.109 \mathrm{E}+06$ & 0.1111 & 25.2 & 25.59 & 15.62 \\
\hline 2 & 5 & 24 & 2.35 & 0.70 & $1.302 E+05$ & $3.255 \mathrm{E}+05$ & 0.4000 & 3.9 & 91.66 & 76.80 \\
\hline 10 & 135 & 35 & 2.73 & 0.83 & $4.464 \mathrm{E}+05$ & $6.027 \mathrm{E}+06$ & 0.0741 & 72.1 & 17.07 & 5.64 \\
\hline 2 & 14 & 18 & 2.31 & 0.60 & $1.736 \mathrm{E}+05$ & $1.215 \mathrm{E}+06$ & 0.1429 & 14.5 & 32.89 & 24.90 \\
\hline 14 & 120 & 60 & 1.99 & 0.47 & $3.646 \mathrm{E}+05$ & $3.125 \mathrm{E}+06$ & 0.1167 & 37.4 & 26.87 & 7.68 \\
\hline 1 & 9 & 24 & 1.79 & 0.48 & $6.510 \mathrm{E}+04$ & $5.859 E+05$ & 0.1111 & 7.0 & 25.59 & 27.00 \\
\hline & (8) & 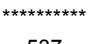 & 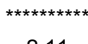 & 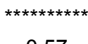 & $* * * * * * * * * *$ & $* * * * * * * * * *$ & $* * * * \star * * * * *$ & $* * * \star * * * * \star * *$ & 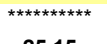 & 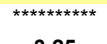 \\
\hline 75 & 687 & 537 & 2.11 & 0.57 & $2.182 \mathrm{E}+05$ & $1.999 \mathrm{E}+06$ & 0.1092 & 23.9 & 25.15 & 3.25 \\
\hline Pooled Ratio & & 0.1092 & \pm & 0.0141 & & & & & & \\
\hline Mean Ratio & & 0.1368 & \pm & 0.0188 & & & & & & \\
\hline Pooled Age & & 25.15 & \pm & 3.25 & 1 S.E. & & & & & \\
\hline Mean Crystal Ag & & 31.49 & \pm & 4.35 & 1 S.E. & & & & & \\
\hline Binomial Age & & 25.38 & + & 6.55 & "+95\%" & & & & & \\
\hline & & & - & 5.83 & "-95\%" & & & & & \\
\hline Central Age & & 25.15 & \pm & 3.25 & & & & & & \\
\hline Age Dispersion & & & & & & & & & & \\
\hline Chi-squared & & 5.585 & with & 15 & \multirow{2}{*}{\multicolumn{6}{|c|}{ degrees of freedom }} \\
\hline$P($ Chi-Sq) & & 98.59 & & & & & & & & \\
\hline MSWD & & 0. & & & & & & & & \\
\hline
\end{tabular}


Table S2

\begin{tabular}{|c|c|c|c|c|c|c|c|c|c|c|}
\hline \multirow{2}{*}{\multicolumn{2}{|c|}{$\begin{array}{l}\text { Sample Number } \\
\text { Position (\#) }\end{array}$}} & \\
\hline & & \multicolumn{9}{|l|}{ DER-2 } \\
\hline \multirow{2}{*}{\multicolumn{2}{|c|}{$\begin{array}{l}\text { Area of Graticule Square } \\
\text { No of Crystals }\end{array}$}} & \multicolumn{9}{|l|}{$6.400 \mathrm{E}-07$} \\
\hline & & \multicolumn{9}{|l|}{20} \\
\hline \multicolumn{2}{|c|}{$\begin{array}{l}\text { No. of Crystals } \\
\text { Zeta Factor } \pm \text { Error }\end{array}$} & 368.1 & \multicolumn{8}{|l|}{14.9} \\
\hline \multicolumn{2}{|c|}{ Rho d (\% Relative Error) } & $1.215 \mathrm{E}+06$ & \multirow{2}{*}{\multicolumn{8}{|c|}{1.60}} \\
\hline & 3889 & & & & & & & & \\
\hline $\mathrm{Ns}$ & $\mathrm{Ni}$ & $\mathrm{Ng}$ & Dpar & Dper & $\rho s$ & $\rho \mathrm{i}$ & $\rho s / \rho i$ & Uppm & Age (Ma) & Age error \\
\hline 4 & 3 & 70 & 2.86 & 0.76 & $8.929 \mathrm{E}+04$ & $6.696 \mathrm{E}+04$ & 1.3333 & 0.8 & 291.47 & 222.98 \\
\hline 3 & 8 & 40 & 2.43 & 0.68 & $1.172 E+05$ & $3.125 \mathrm{E}+05$ & 0.3750 & 3.9 & 83.32 & 56.52 \\
\hline 1 & 11 & 80 & 2.68 & 0.68 & $1.953 \mathrm{E}+04$ & $2.148 \mathrm{E}+05$ & 0.0909 & 2.7 & 20.30 & 21.22 \\
\hline 10 & 25 & 100 & 2.80 & 0.73 & $1.563 \mathrm{E}+05$ & $3.906 \mathrm{E}+05$ & 0.4000 & 4.8 & 88.83 & 33.46 \\
\hline 4 & 15 & 60 & 2.74 & 0.56 & $1.042 E+05$ & $3.906 \mathrm{E}+05$ & 0.2667 & 4.8 & 59.36 & 33.50 \\
\hline 10 & 42 & 50 & 2.39 & 0.51 & $3.125 \mathrm{E}+05$ & $1.313 \mathrm{E}+06$ & 0.2381 & 16.2 & 53.02 & 18.80 \\
\hline 7 & 28 & 60 & 2.26 & 0.52 & $1.823 \mathrm{E}+05$ & $7.292 \mathrm{E}+05$ & 0.2500 & 9.0 & 55.66 & 23.65 \\
\hline 10 & 20 & 80 & 2.46 & 0.74 & $1.953 E+05$ & $3.906 \mathrm{E}+05$ & 0.5000 & 4.8 & 110.85 & 43.20 \\
\hline 6 & 26 & 100 & 2.43 & 0.62 & $9.375 \mathrm{E}+04$ & $4.063 E+05$ & 0.2308 & 5.0 & 51.40 & 23.39 \\
\hline 4 & 7 & 35 & 2.10 & 0.59 & $1.786 \mathrm{E}+05$ & $3.125 \mathrm{E}+05$ & 0.5714 & 3.9 & 126.53 & 79.50 \\
\hline 5 & 16 & 80 & 2.82 & 0.78 & $9.766 \mathrm{E}+04$ & $3.125 E+05$ & 0.3125 & 3.9 & 69.51 & 35.74 \\
\hline 6 & 38 & 100 & 2.25 & 0.83 & $9.375 \mathrm{E}+04$ & $5.938 \mathrm{E}+05$ & 0.1579 & 7.3 & 35.21 & 15.54 \\
\hline 52 & 126 & 50 & 2.69 & 0.74 & $1.625 \mathrm{E}+06$ & $3.938 \mathrm{E}+06$ & 0.4127 & 48.6 & 91.63 & 15.62 \\
\hline 3 & 10 & 50 & 2.53 & 0.76 & $9.375 \mathrm{E}+04$ & $3.125 \mathrm{E}+05$ & 0.3000 & 3.9 & 66.74 & 44.03 \\
\hline 12 & 20 & 100 & 2.54 & 0.73 & $1.875 \mathrm{E}+05$ & $3.125 E+05$ & 0.6000 & 3.9 & 132.80 & 48.83 \\
\hline 5 & 17 & 40 & 2.12 & 0.67 & $1.953 \mathrm{E}+05$ & $6.641 \mathrm{E}+05$ & 0.2941 & 8.2 & 65.44 & 33.41 \\
\hline 39 & 43 & 100 & 2.14 & 0.82 & $6.094 \mathrm{E}+05$ & $6.719 \mathrm{E}+05$ & 0.9070 & 8.3 & 199.69 & 45.01 \\
\hline 9 & 17 & 70 & 2.31 & 0.60 & $2.009 \mathrm{E}+05$ & $3.795 \mathrm{E}+05$ & 0.5294 & 4.7 & 117.31 & 48.63 \\
\hline 12 & 24 & 100 & 2.55 & 0.66 & $1.875 \mathrm{E}+05$ & $3.750 \mathrm{E}+05$ & 0.5000 & 4.6 & 110.85 & 39.49 \\
\hline 16 & 45 & 70 & 2.48 & 0.60 & $3.571 \mathrm{E}+05$ & $1.004 \mathrm{E}+06$ & 0.3556 & 12.4 & 79.02 & 23.26 \\
\hline 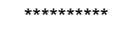 & 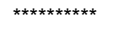 & 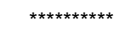 & 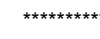 & $* * * \star * \star * * \star *$ & 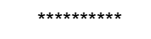 & 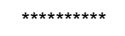 & 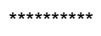 & 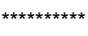 & 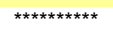 & 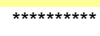 \\
\hline 218 & 541 & 1435 & 2.48 & 0.68 & $2.374 \mathrm{E}+05$ & $5.891 \mathrm{E}+05$ & 0.4030 & 7.3 & 89.49 & 8.17 \\
\hline \multicolumn{2}{|c|}{ Pooled Ratio } & 0.4030 & \pm & 0.0368 & & & & & & \\
\hline \multicolumn{2}{|c|}{ Mean Ratio } & 0.4313 & \pm & 0.0627 & & & & & & \\
\hline \multicolumn{2}{|c|}{ Pooled Age } & 89.49 & \pm & 8.17 & 1 S.E. & & & & & \\
\hline \multirow{2}{*}{\multicolumn{2}{|c|}{$\begin{array}{l}\text { Mean Crystal Age } \\
\text { Binomial Age }\end{array}$}} & 95.73 & \pm & 14.01 & 1S.E. & & & & & \\
\hline & & 89.81 & + & 14.98 & "+95\%" & & & & & \\
\hline \multicolumn{2}{|c|}{ Binomial Age } & & - & 13.62 & "-95\%" & & & & & \\
\hline \multicolumn{2}{|c|}{ Central Age } & 86.21 & \pm & 10.70 & & & & & & \\
\hline \multicolumn{2}{|c|}{ Age Dispersion } & \multicolumn{2}{|c|}{$32.32 \%$} & & & & & & & \\
\hline \multicolumn{2}{|c|}{ Chi-squared } & \multirow{3}{*}{\multicolumn{2}{|c|}{$\begin{array}{l}33.693 \\
2.00 \%\end{array}$}} & 19 & degrees of free & dom & & & & \\
\hline$P($ Chi-Sq) & & & & & & & & & & \\
\hline MSWD & & & & & & & & & & \\
\hline
\end{tabular}

\begin{tabular}{|c|c|c|}
\hline Length & Angle & Dpar \\
\hline 10.87 & 63 & 2.46 \\
\hline 12.29 & 23 & 2.46 \\
\hline 11.13 & 89 & 2.46 \\
\hline 11.69 & 13 & 2.46 \\
\hline 13.91 & 32 & 1.70 \\
\hline 13.82 & 59 & 3.07 \\
\hline 8.69 & 74 & 3.07 \\
\hline 10.82 & 10 & 2.49 \\
\hline \multicolumn{2}{|c|}{ Mean track length } & $11.65 \pm 0.65$ \\
\hline SD & & 1.71 \\
\hline Skew & & -0.1988 \\
\hline
\end{tabular}


Table S3

\begin{tabular}{|c|c|c|c|c|c|c|c|c|c|c|}
\hline \multicolumn{2}{|l|}{ Sample Number } & \multicolumn{9}{|l|}{ KIRK-1 } \\
\hline \multirow{2}{*}{\multicolumn{2}{|c|}{ Position (\#) }} & 7 & & & & & & & & \\
\hline & & $6.400 \mathrm{E}-07$ & & & & & & & & \\
\hline \multicolumn{2}{|c|}{$\begin{array}{l}\text { Area of Graticule Square } \\
\text { No. of Crystals }\end{array}$} & 12 & & & & & & & & \\
\hline \multicolumn{2}{|c|}{ Zeta Factor \pm Error } & 368.1 & 14.9 & & & & & & & \\
\hline \multicolumn{2}{|c|}{ Rho d (\% Relative Error) } & $1.235 \mathrm{E}+06 \quad 1$ & 1.59 & & & & & & & \\
\hline \multicolumn{2}{|l|}{$\mathrm{Nd}$} & 3951 & & & & & & & & \\
\hline $\mathrm{Ns}$ & $\mathrm{Ni}$ & $\mathrm{Ng}$ & Dpar & Dper & $\rho s$ & $\rho i$ & $\rho s / \rho i$ & Uppm & Age (Ma) & Age error \\
\hline 1 & 13 & 15 & 2.14 & 0.55 & $1.042 \mathrm{E}+05$ & $1.354 \mathrm{E}+06$ & 0.0769 & 16.4 & 17.46 & 18.14 \\
\hline 3 & 21 & 12 & 2.35 & 0.66 & $3.906 \mathrm{E}+05$ & $2.734 E+06$ & 0.1429 & 33.2 & 32.39 & 20.04 \\
\hline 14 & 121 & 16 & 1.97 & 0.59 & $1.367 \mathrm{E}+06$ & $1.182 \mathrm{E}+07$ & 0.1157 & 143.5 & 26.25 & 7.50 \\
\hline 2 & 14 & 18 & 2.13 & 0.49 & $1.736 \mathrm{E}+05$ & $1.215 E+06$ & 0.1429 & 14.8 & 32.39 & 24.53 \\
\hline 2 & 23 & 25 & 2.29 & 0.62 & $1.250 \mathrm{E}+05$ & $1.438 \mathrm{E}+06$ & 0.0870 & 17.5 & 19.74 & 14.57 \\
\hline 3 & 37 & 32 & 2.08 & 0.57 & $1.465 E+05$ & $1.807 E+06$ & 0.0811 & 21.9 & 18.40 & 11.08 \\
\hline 2 & 20 & 10 & 1.71 & 0.64 & $3.125 \mathrm{E}+05$ & $3.125 \mathrm{E}+06$ & 0.1000 & 38.0 & 22.69 & 16.86 \\
\hline 2 & 15 & 15 & 1.98 & 0.41 & $2.083 E+05$ & $1.563 E+06$ & 0.1333 & 19.0 & 30.24 & 22.80 \\
\hline 1 & 7 & 14 & 2.02 & 0.54 & $1.116 \mathrm{E}+05$ & $7.813 E+05$ & 0.1429 & 9.5 & 32.39 & 34.66 \\
\hline 1 & 14 & 9 & 1.99 & 0.58 & $1.736 \mathrm{E}+05$ & $2.431 \mathrm{E}+06$ & 0.0714 & 29.5 & 16.22 & 16.80 \\
\hline 2 & 24 & 20 & 2.46 & 0.62 & $1.563 \mathrm{E}+05$ & $1.875 \mathrm{E}+06$ & 0.0833 & 22.8 & 18.91 & 13.94 \\
\hline 1 & 15 & 20 & 1.98 & 0.74 & $7.813 \mathrm{E}+04$ & $1.172 E+06$ & 0.0667 & 14.2 & 15.14 & 15.65 \\
\hline 34 & 324 & 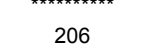 & 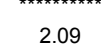 & 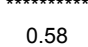 & $2.579 \mathrm{E}+05$ & $2.458 \mathrm{E}+06$ & 0.1049 & 29.8 & 23.81 & 4.42 \\
\hline Pooled Ratio & & 0.1049 & \pm & 0.0195 & & & & & & \\
\hline Mean Ratio & & 0.1037 & \pm & 0.0087 & & & & & & \\
\hline Pooled Age & & 23.81 & \pm & 4.42 & 1 S.E. & & & & & \\
\hline Mean Crystal Ag & & 23.52 & \pm & 1.98 & 1 S.E. & & & & & \\
\hline Binomial Age & & 24.30 & + & 9.63 & "+95\%" & & & & & \\
\hline & & & - & 8.08 & "-95\%" & & & & & \\
\hline Central Age & & 23.81 & \pm & 4.42 & & & & & & \\
\hline Age Dispersion & & $0.00 \%$ & & & & & & & & \\
\hline Chi-squared & & 1.505 & with & 11 & degrees of free & dom & & & & \\
\hline$P($ Chi-Sq) & & $99.96 \%$ & & & & & & & & \\
\hline MSWD & & 0.14 & & & & & & & & \\
\hline
\end{tabular}


Table S4

\begin{tabular}{|c|c|c|c|c|c|c|c|c|c|c|}
\hline \multirow{2}{*}{\multicolumn{2}{|c|}{$\begin{array}{l}\text { Sample Number } \\
\text { Position (\#) }\end{array}$}} & \multicolumn{9}{|l|}{ KOP-6 } \\
\hline & & 10 & & & & & & & & \\
\hline \multirow{2}{*}{\multicolumn{2}{|c|}{$\begin{array}{l}\text { Area of Graticule Square } \\
\text { No of Crystals }\end{array}$}} & $6.400 \mathrm{E}-07$ & & & & & & & & \\
\hline & & 20 & & & & & & & & \\
\hline \multicolumn{2}{|c|}{$\begin{array}{l}\text { No. of Crystals } \\
\text { Zeta Factor } \pm \text { Error }\end{array}$} & 368.1 & \multicolumn{8}{|l|}{14.9} \\
\hline \multicolumn{2}{|c|}{ Rho d (\% Relative Error) } & $1.206 \mathrm{E}+06$ & \multicolumn{8}{|l|}{1.61} \\
\hline \multicolumn{2}{|c|}{$\mathrm{Nd}$} & \\
\hline $\mathrm{Ns}$ & $\mathrm{Ni}$ & $\mathrm{Ng}$ & Dpar & Dper & $\rho s$ & $\rho \mathrm{i}$ & $\rho s / \rho i$ & Uppm & Age (Ma) & Age error \\
\hline 9 & 108 & 60 & 1.87 & 0.48 & $2.344 \mathrm{E}+05$ & $2.813 E+06$ & 0.0833 & 35.0 & 18.47 & 6.46 \\
\hline 3 & 38 & 30 & 2.04 & 0.67 & $1.563 \mathrm{E}+05$ & $1.979 E+06$ & 0.0789 & 24.6 & 17.50 & 10.52 \\
\hline 6 & 49 & 70 & 2.03 & 0.73 & $1.339 \mathrm{E}+05$ & $1.094 \mathrm{E}+06$ & 0.1224 & 13.6 & 27.12 & 11.79 \\
\hline 2 & 22 & 36 & 2.25 & 0.74 & $8.681 \mathrm{E}+04$ & $9.549 \mathrm{E}+05$ & 0.0909 & 11.9 & 20.15 & 14.91 \\
\hline 3 & 34 & 35 & 2.20 & 0.46 & $1.339 \mathrm{E}+05$ & $1.518 \mathrm{E}+06$ & 0.0882 & 18.9 & 19.56 & 11.81 \\
\hline 3 & 36 & 49 & 2.27 & 0.63 & $9.566 \mathrm{E}+04$ & $1.148 \mathrm{E}+06$ & 0.0833 & 14.3 & 18.47 & 11.13 \\
\hline 3 & 42 & 36 & 2.34 & 0.56 & $1.302 E+05$ & $1.823 \mathrm{E}+06$ & 0.0714 & 22.7 & 15.84 & 9.49 \\
\hline 7 & 64 & 32 & 2.11 & 0.44 & $3.418 \mathrm{E}+05$ & $3.125 \mathrm{E}+06$ & 0.1094 & 38.9 & 24.23 & 9.70 \\
\hline 2 & 14 & 21 & 1.83 & 0.60 & $1.488 \mathrm{E}+05$ & $1.042 \mathrm{E}+06$ & 0.1429 & 13.0 & 31.63 & 23.95 \\
\hline 7 & 62 & 40 & 1.88 & 0.65 & $2.734 E+05$ & $2.422 \mathrm{E}+06$ & 0.1129 & 30.1 & 25.01 & 10.03 \\
\hline 9 & 74 & 32 & 2.09 & 0.45 & $4.395 \mathrm{E}+05$ & $3.613 \mathrm{E}+06$ & 0.1216 & 44.9 & 26.94 & 9.58 \\
\hline 7 & 46 & 18 & 2.09 & 0.51 & $6.076 \mathrm{E}+05$ & $3.993 \mathrm{E}+06$ & 0.1522 & 49.7 & 33.69 & 13.75 \\
\hline 4 & 29 & 20 & 1.99 & 0.66 & $3.125 \mathrm{E}+05$ & $2.266 \mathrm{E}+06$ & 0.1379 & 28.2 & 30.54 & 16.35 \\
\hline 5 & 53 & 35 & 2.13 & 0.55 & $2.232 E+05$ & $2.366 \mathrm{E}+06$ & 0.0943 & 29.4 & 20.91 & 9.82 \\
\hline 11 & 131 & 48 & 2.10 & 0.65 & $3.581 \mathrm{E}+05$ & $4.264 E+06$ & 0.0840 & 53.0 & 18.61 & 5.90 \\
\hline 3 & 38 & 25 & 1.86 & 0.72 & $1.875 \mathrm{E}+05$ & $2.375 E+06$ & 0.0789 & 29.5 & 17.50 & 10.52 \\
\hline 6 & 50 & 35 & 1.67 & 0.36 & $2.679 \mathrm{E}+05$ & $2.232 \mathrm{E}+06$ & 0.1200 & 27.8 & 26.58 & 11.54 \\
\hline 19 & 218 & 20 & 2.11 & 0.51 & $1.484 \mathrm{E}+06$ & $1.703 \mathrm{E}+07$ & 0.0872 & 211.8 & 19.32 & 4.70 \\
\hline 3 & 34 & 100 & 1.98 & 0.59 & $4.688 \mathrm{E}+04$ & $5.313 E+05$ & 0.0882 & 6.6 & 19.56 & 11.81 \\
\hline 6 & 51 & 15 & 1.92 & 0.72 & $6.250 \mathrm{E}+05$ & $5.313 E+06$ & 0.1176 & 66.1 & 26.06 & 11.30 \\
\hline & & 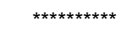 & 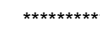 & 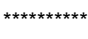 & $\star \star * \star * \star * \star * \star * * *$ & 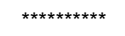 & 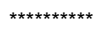 & 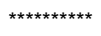 & 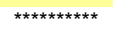 & \\
\hline 118 & 1193 & 757 & 2.04 & 0.58 & $2.436 \mathrm{E}+05$ & $2.462 E+06$ & 0.0989 & 30.6 & 21.92 & 2.32 \\
\hline \multicolumn{2}{|c|}{ Pooled Ratio } & 0.0989 & \pm & 0.0105 & & & & & & \\
\hline \multicolumn{2}{|c|}{ Mean Ratio } & 0.1033 & \pm & 0.0053 & & & & & & \\
\hline \multicolumn{2}{|c|}{ Pooled Age } & 21.92 & \pm & 2.32 & 1 S.E. & & & & & \\
\hline \multirow{2}{*}{\multicolumn{2}{|c|}{$\begin{array}{l}\text { Mean Crystal Age } \\
\text { Binomial Age }\end{array}$}} & 22.89 & \pm & 1.18 & 1 S.E. & & & & & \\
\hline & & 22.05 & + & 4.44 & "+95\%" & & & & & \\
\hline & & - & 4.06 & "-95\%" & & & & & \\
\hline \multicolumn{2}{|c|}{ Central Age } & 21.92 & \pm & 2.32 & & & & & & \\
\hline \multicolumn{2}{|c|}{ Age Dispersion } & \multicolumn{2}{|c|}{$0.00 \%$} & & & & & & & \\
\hline \multicolumn{2}{|c|}{ Chi-squared } & \multicolumn{2}{|c|}{4.459 with } & 19 & degrees of free & dom & & & & \\
\hline \multirow{2}{*}{\multicolumn{2}{|c|}{$\begin{array}{l}\text { P (Chi-Sq) } \\
\text { MSWD }\end{array}$}} & \multirow{2}{*}{\multicolumn{2}{|c|}{$\begin{array}{l}99.98 \% \\
0.23\end{array}$}} & & & & & & & \\
\hline & & & & & & & & & & \\
\hline
\end{tabular}


Table S5

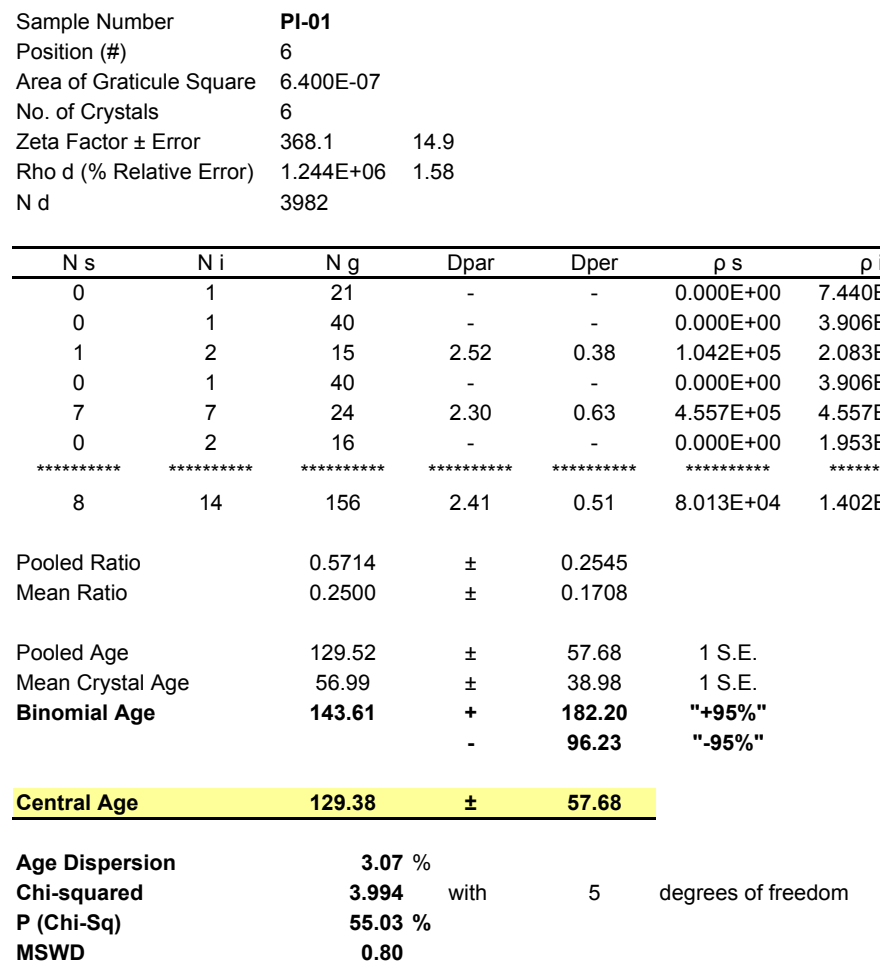


Table S6

Sample Number $\quad$ SEY1

Sample Numbe

Area of Graticule Square $6.400 \mathrm{E}-07$

No. of Crystals 13

$\begin{array}{lll} & & \\ 2 \text { eta Factor } \pm \text { Error } & 368.1 & 14.9\end{array}$

$\begin{array}{lll}\text { Rho d (\% Relative Error) } & 1.274 E+06 & 1.57\end{array}$

$\mathrm{Nd} \quad 4076$

\begin{tabular}{|c|c|c|c|c|c|c|c|c|c|c|}
\hline $\mathrm{Ns}$ & $\mathrm{Ni}$ & $\mathrm{Ng}$ & Dpar & Dper & $\rho s$ & $\rho i$ & $\rho s / \rho i$ & Uppm & Age (Ma) & Age error \\
\hline 2 & 16 & 25 & 1.90 & 0.60 & $1.250 \mathrm{E}+05$ & $1.000 \mathrm{E}+06$ & 0.1250 & 11.8 & 29.24 & 21.97 \\
\hline 4 & 20 & 16 & 1.92 & 0.58 & $3.906 \mathrm{E}+05$ & $1.953 E+06$ & 0.2000 & 23.0 & 46.73 & 25.67 \\
\hline 1 & 5 & 40 & 1.98 & 0.48 & $3.906 \mathrm{E}+04$ & $1.953 E+05$ & 0.2000 & 2.3 & 46.73 & 51.23 \\
\hline 1 & 12 & 60 & 2.61 & 0.96 & $2.604 \mathrm{E}+04$ & $3.125 \mathrm{E}+05$ & 0.0833 & 3.7 & 19.51 & 20.32 \\
\hline 1 & 4 & 15 & 2.33 & 0.42 & $1.042 \mathrm{E}+05$ & 4.167E+05 & 0.2500 & 4.9 & 58.36 & 65.29 \\
\hline 0 & 1 & 1 & - & - & $0.000 E+00$ & $1.563 \mathrm{E}+06$ & 0.0000 & 18.4 & 0.00 & $n / a$ \\
\hline 1 & 8 & 20 & 2.47 & 0.73 & $7.813 E+04$ & $6.250 \mathrm{E}+05$ & 0.1250 & 7.4 & 29.24 & 31.04 \\
\hline 2 & 8 & 12 & 2.06 & 0.66 & $2.604 E+05$ & $1.042 \mathrm{E}+06$ & 0.2500 & 12.3 & 58.36 & 46.20 \\
\hline 17 & 74 & 40 & 1.85 & 0.56 & $6.641 \mathrm{E}+05$ & $2.891 \mathrm{E}+06$ & 0.2297 & 34.0 & 53.64 & 14.61 \\
\hline 4 & 24 & 25 & 1.71 & 0.44 & $2.500 \mathrm{E}+05$ & $1.500 \mathrm{E}+06$ & 0.1667 & 17.7 & 38.96 & 21.11 \\
\hline 8 & 40 & 35 & 2.04 & 0.40 & $3.571 \mathrm{E}+05$ & $1.786 \mathrm{E}+06$ & 0.2000 & 21.0 & 46.73 & 18.21 \\
\hline 1 & 2 & 24 & 2.60 & 0.72 & $6.510 \mathrm{E}+04$ & $1.302 \mathrm{E}+05$ & 0.5000 & 1.5 & 116.19 & 142.39 \\
\hline 2 & 16 & 15 & 2.13 & 0.56 & $2.083 \mathrm{E}+05$ & $1.667 \mathrm{E}+06$ & 0.1250 & 19.6 & 29.24 & 21.97 \\
\hline 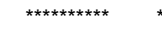 & $* * \star * \star * * * * * * * *$ & 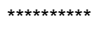 & $* * * * * * * * * * *$ & 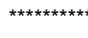 & $* * * * * * * * * * *$ & 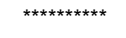 & 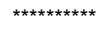 & 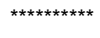 & $* * * * * * * * *$ & 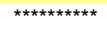 \\
\hline 44 & 230 & 328 & 2.13 & 0.59 & $2.096 \mathrm{E}+05$ & $1.096 \mathrm{E}+06$ & 0.1913 & 12.9 & 44.70 & 7.61 \\
\hline Pooled Ratio & & 0.1913 & \pm & 0.0326 & & & & & & \\
\hline Mean Ratio & & 0.1888 & \pm & 0.0326 & & & & & & \\
\hline Pooled Age & & 44.70 & \pm & 7.61 & 1 S.E. & & & & & \\
\hline Mean Crystal Ag & & 44.12 & \pm & 7.64 & 1 S.E. & & & & & \\
\hline \multirow{2}{*}{\multicolumn{2}{|c|}{ Binomial Age }} & 45.44 & + & 16.41 & "+95\%" & & & & & \\
\hline & & & - & 13.80 & "-95\%" & & & & & \\
\hline Central Age & & 44.70 & \pm & 7.61 & & & & & & \\
\hline Age Dispersion & & & & & & & & & & \\
\hline Chi-squared & & 3.07 & with & 12 & degrees of fre & dom & & & & \\
\hline$P(C h i-S q)$ & & 99.5 & & & & & & & & \\
\hline MSWD & & 0.2 & & & & & & & & \\
\hline
\end{tabular}


Table S7

\begin{tabular}{|c|c|c|c|c|c|c|}
\hline \multirow{2}{*}{\multicolumn{2}{|c|}{$\begin{array}{l}\text { Sample Number } \\
\text { Position (\#) }\end{array}$}} & \\
\hline & & $\begin{array}{l}\text { SEY3 } \\
4\end{array}$ & & & & \\
\hline \multirow{2}{*}{\multicolumn{2}{|c|}{$\begin{array}{l}\text { Position (\#) } \\
\text { Area of Graticule Square } \\
\text { No. of Crystals }\end{array}$}} & $6.400 \mathrm{E}-07$ & & & & \\
\hline & & 20 & & & & \\
\hline \multicolumn{2}{|c|}{ Zeta Factor \pm Error } & 368.1 & 14.9 & & & \\
\hline \multicolumn{2}{|c|}{ Rho d (\% Relative Error) } & $1.264 E+06$ & 1.57 & & & \\
\hline \multicolumn{2}{|l|}{$\mathrm{Nd}$} & 4045 & & & & \\
\hline $\mathrm{Ns}$ & $\mathrm{Ni}$ & $\mathrm{Ng}$ & Dpar & Dper & $\rho s$ & \\
\hline 12 & 43 & 42 & 2.50 & 0.66 & $4.464 \mathrm{E}+05$ & 1.60 \\
\hline 1 & 1 & 24 & 2.36 & 0.46 & $6.510 \mathrm{E}+04$ & 6.51 \\
\hline 3 & 8 & 30 & 2.60 & 0.72 & $1.563 E+05$ & 4.16 \\
\hline 2 & 4 & 70 & 2.50 & 0.54 & $4.464 E+04$ & 8.92 \\
\hline 22 & 50 & 20 & 1.94 & 0.63 & $1.719 E+06$ & 3.90 \\
\hline 2 & 5 & 40 & 1.83 & 0.77 & $7.813 E+04$ & 1.95 \\
\hline 4 & 7 & 35 & 2.85 & 0.66 & $1.786 \mathrm{E}+05$ & 3.12 \\
\hline 7 & 31 & 36 & 2.02 & 0.49 & $3.038 \mathrm{E}+05$ & 1.34 \\
\hline 2 & 15 & 50 & 2.59 & 0.66 & $6.250 E+04$ & 4.68 \\
\hline 6 & 23 & 25 & 2.42 & 0.49 & $3.750 E+05$ & 1.43 \\
\hline 7 & 14 & 70 & 2.80 & 0.84 & $1.563 E+05$ & 3.12 \\
\hline 6 & 10 & 60 & 2.32 & 0.70 & $1.563 E+05$ & 2.60 \\
\hline 11 & 30 & 80 & 2.63 & 0.63 & $2.148 \mathrm{E}+05$ & 5.85 \\
\hline 5 & 23 & 50 & 2.42 & 0.66 & $1.563 E+05$ & 7.18 \\
\hline 8 & 30 & 80 & 2.62 & 0.49 & $1.563 E+05$ & 5.85 \\
\hline 6 & 30 & 100 & 2.78 & 0.80 & $9.375 E+04$ & 4.68 \\
\hline 3 & 10 & 50 & 2.42 & 0.72 & $9.375 E+04$ & 3.12 \\
\hline 4 & 8 & 50 & 2.53 & 0.71 & $1.250 \mathrm{E}+05$ & 2.50 \\
\hline 5 & 12 & 64 & 2.48 & 0.72 & $1.221 \mathrm{E}+05$ & 2.93 \\
\hline 4 & 26 & 100 & 2.79 & 0.69 & $6.250 E+04$ & 4.06 \\
\hline & & & & & 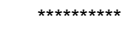 & \\
\hline 120 & 380 & 1076 & 2.47 & 0.65 & $1.743 E+05$ & 5.51 \\
\hline \multicolumn{2}{|l|}{ Pooled Ratio } & 0.3158 & \pm & 0.0358 & & \\
\hline \multicolumn{2}{|l|}{ Mean Ratio } & 0.3853 & \pm & 0.0446 & & \\
\hline \multicolumn{2}{|l|}{ Pooled Age } & 73.05 & \pm & 8.28 & 1 S.E. & \\
\hline \multirow{2}{*}{\multicolumn{2}{|c|}{$\begin{array}{l}\text { Mean Crystal Age } \\
\text { Binomial Age }\end{array}$}} & 89.03 & \pm & 10.37 & $1 \mathrm{~S} . \mathrm{E}$. & \\
\hline & & 73.52 & + & 16.28 & "+95\%" & \\
\hline & & & - & 14.46 & "-95\%" & \\
\hline \multicolumn{2}{|l|}{ Central Age } & 73.05 & \pm & 8.28 & & \\
\hline \multirow{2}{*}{\multicolumn{2}{|c|}{$\begin{array}{l}\text { Age Dispersion } \\
\text { Chi-squared }\end{array}$}} & \multicolumn{2}{|c|}{$0.19 \%$} & & & \\
\hline & & \multirow{2}{*}{\multicolumn{2}{|c|}{$\begin{array}{l}13.479 \\
81.32 \%\end{array}$}} & 19 & degrees of fre & dom \\
\hline \multicolumn{2}{|l|}{ Chi-squared } & & & & & \\
\hline \multicolumn{2}{|l|}{$\begin{array}{l}\text { MSWD } \\
\text { MSI }\end{array}$} & \multicolumn{2}{|c|}{0.71} & & & \\
\hline
\end{tabular}


Table S8

Sample Number SEY3

Area of Graticule Square $\quad 6.400 \mathrm{E}-07$

20

$\begin{array}{lll} & 20.1 & \end{array}$

$\begin{array}{lll} & 1.264 E+06 & 1.57\end{array}$

$\mathrm{Nd}$

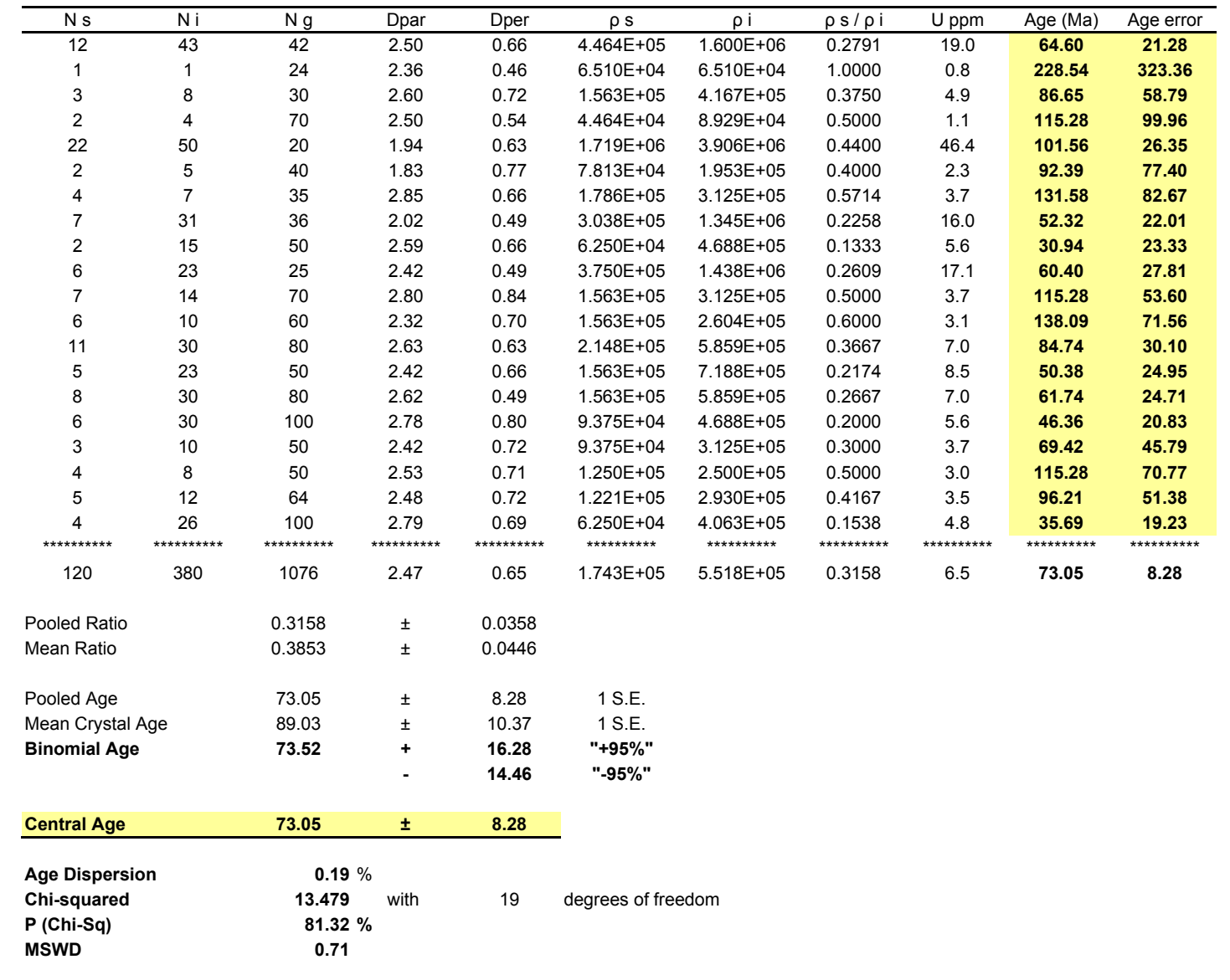




\section{Table S9:}

Vitrinite reflectance data from Hokerek et al., (2014).

\begin{tabular}{lc}
\hline Sample \# & Ro (\%) \\
\hline KS08B & 2.7 \\
KS15 & 2.2 \\
KS18 & 2.7 \\
KR01 & 2.5 \\
KR02 & 2.6 \\
KR03B & 2.4 \\
KR04A & 2.3 \\
KR07 & 2.6 \\
KR08 & 2.5 \\
AVERAGE & $\mathbf{2 . 5}$ \\
\hline 30 & $\mathbf{0 . 1 7}$
\end{tabular}

Hokerek, S., Unal, N., Altunsoy, M., Ozcelik, O., \& Kuscu, M. (2014).

Organic Facies Characteristics of the Triassic Kasımlar Formation, Anamas-Akseki Platform, Western Taurides, Turkey. Energy Procedia, 59, 150-157.

http://doi.org/10.1016/j.egypro.2014.10.361 


\section{Figure S4: KOP-6}

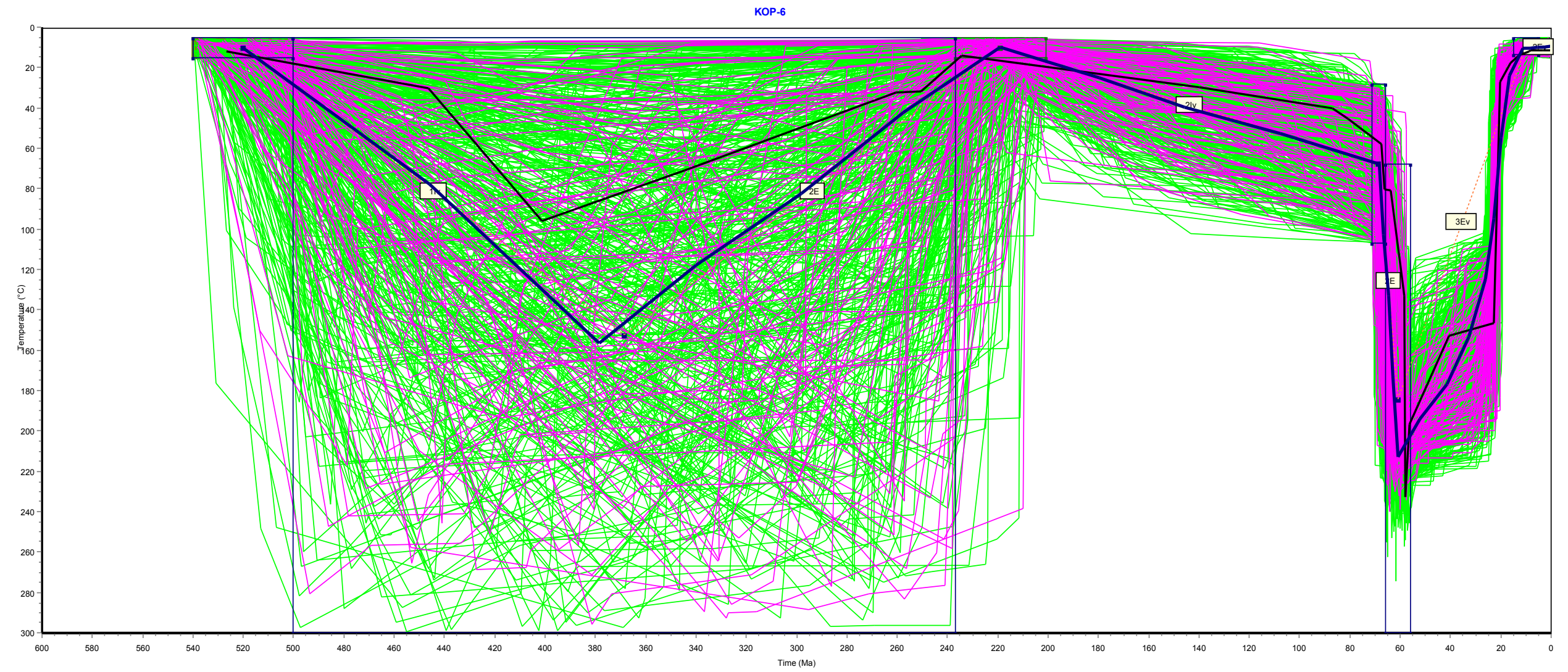

\begin{tabular}{|c|c|c|c|c|c|c|c|}
\hline & & \multicolumn{2}{|l|}{$\mathrm{AHe}$} & \multicolumn{2}{|l|}{ AFT } & \multicolumn{2}{|c|}{ Vro } \\
\hline & & Measured age & Model age & Measured age & Model age & Measured & Model \\
\hline Simulated paths & 106719 & $21.8 \pm 1.5$ & 20.9 & $21.9 \pm 2.3$ & 22.7 & $2.50 \pm 0.34$ & 2.47 \\
\hline Acceptable & 463 & $20.9 \pm 1.5$ & 20.8 & & & & \\
\hline Good & 100 & & & & & & \\
\hline
\end{tabular}


Figure S4: KIRK-1

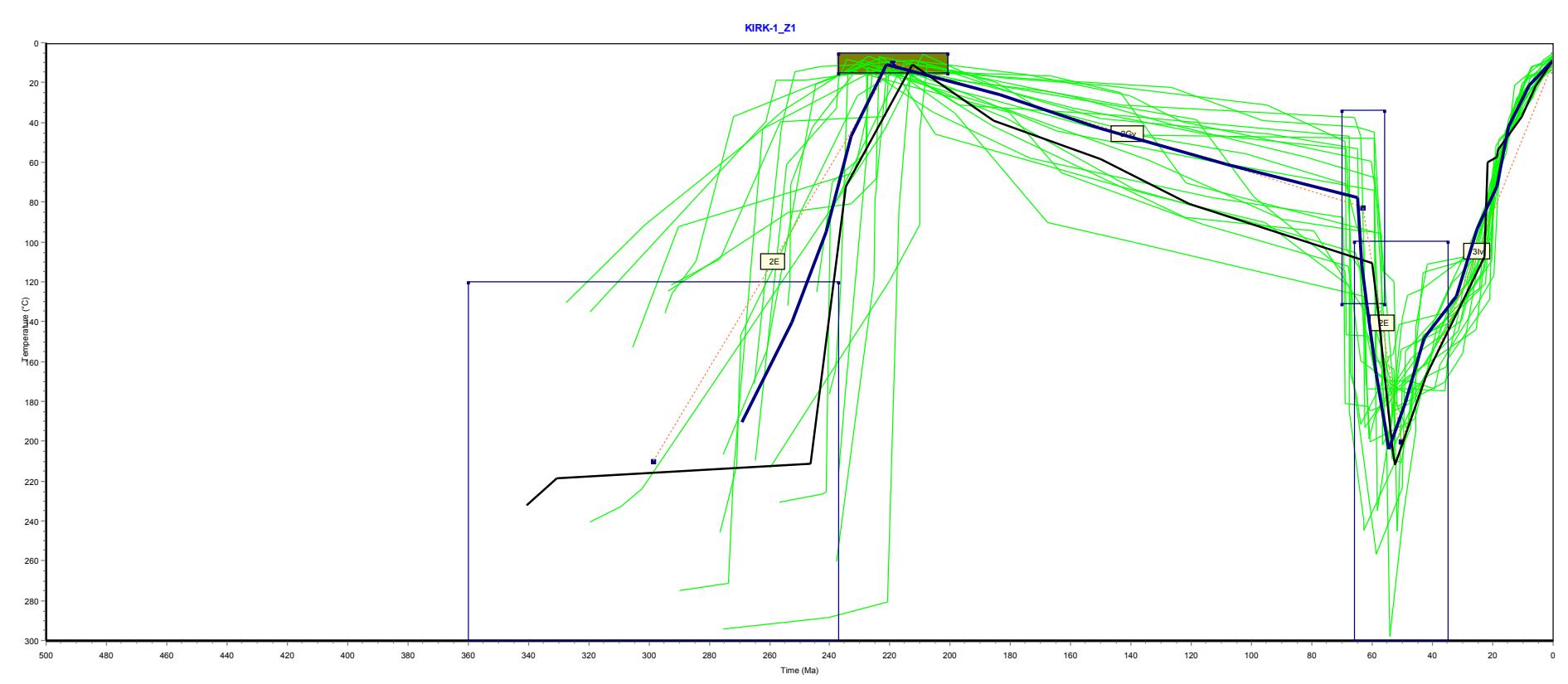

\begin{tabular}{ll}
\multicolumn{2}{c}{ KIRK-1 Zr1 } \\
\hline Simulated paths & 717644 \\
Acceptable & 21 \\
Good & 0 \\
& \\
AHe & \\
Measured age & Model age \\
\hline $16.6 \pm 2$ & 13.6 \\
$20.2 \pm 1.6$ & 16.5 \\
$14.7 \pm 1.1$ & 16.1 \\
$14.7 \pm 1.1$ & 16.5 \\
$19.6 \pm 1.5$ & 16.5
\end{tabular}

AFT

Measured age Model age $23.8 \pm 4.4$ 37.7

$\mathrm{ZHe}$

Measured age Model age $52.2 \pm 4.3$ 43.6

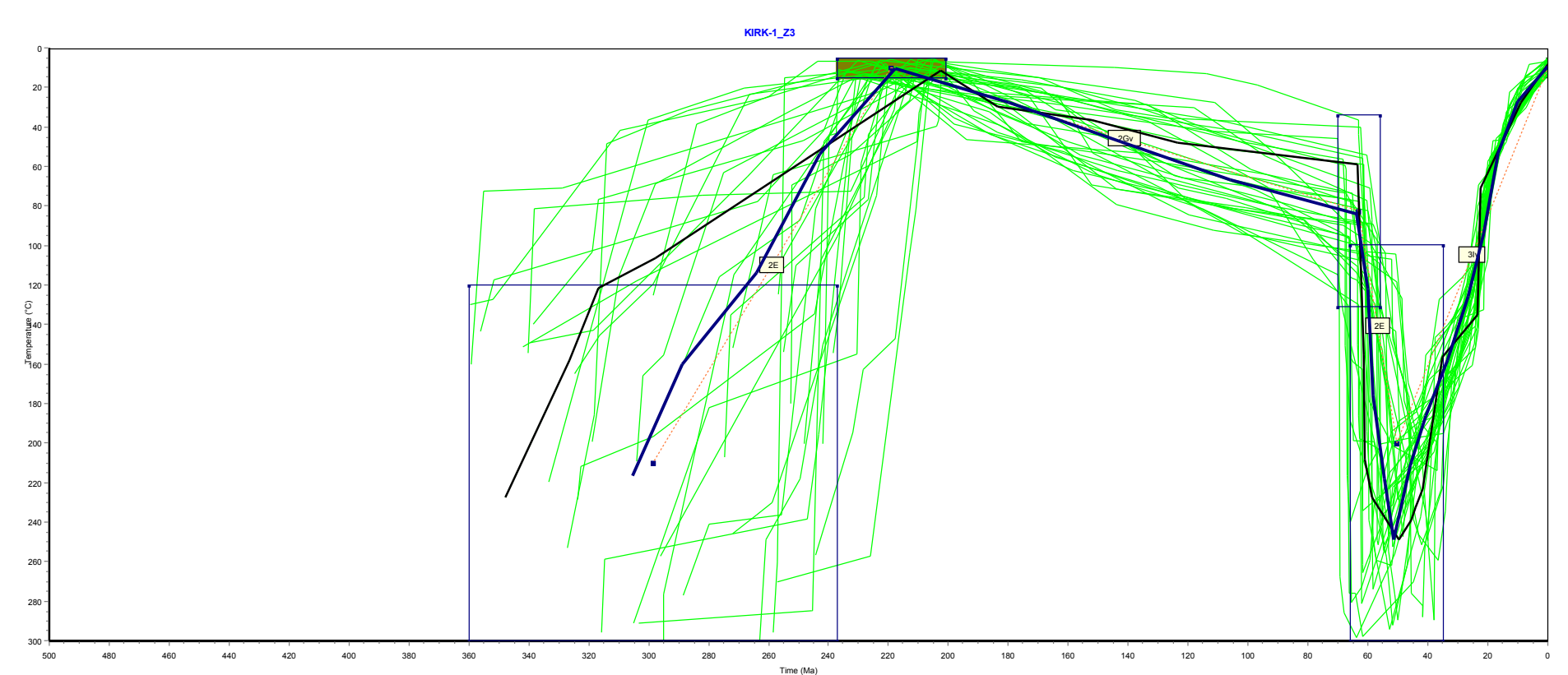

\begin{tabular}{ll}
\multicolumn{2}{c}{ KIRK-1 Zr2 } \\
\hline Simulated paths & 316413 \\
Acceptable & 35 \\
Good & 0 \\
& \\
AHe & \\
Measured age & Model age \\
\hline $16.6 \pm 2$ & 14 \\
$20.2 \pm 1.6$ & 16.4 \\
$14.7 \pm 1.1$ & 16.1 \\
$14.7 \pm 1.1$ & 16.3 \\
$19.6 \pm 1.5$ & 16.5 \\
AFT & \\
Measured age & Model age \\
\hline $23.8 \pm 4.4$ & 32.4 \\
ZHe & \\
Measured age & Model age \\
\hline $38.1 \pm 3.1$ & 37.5
\end{tabular}




\section{Figure S5: BA-1}

BA-1

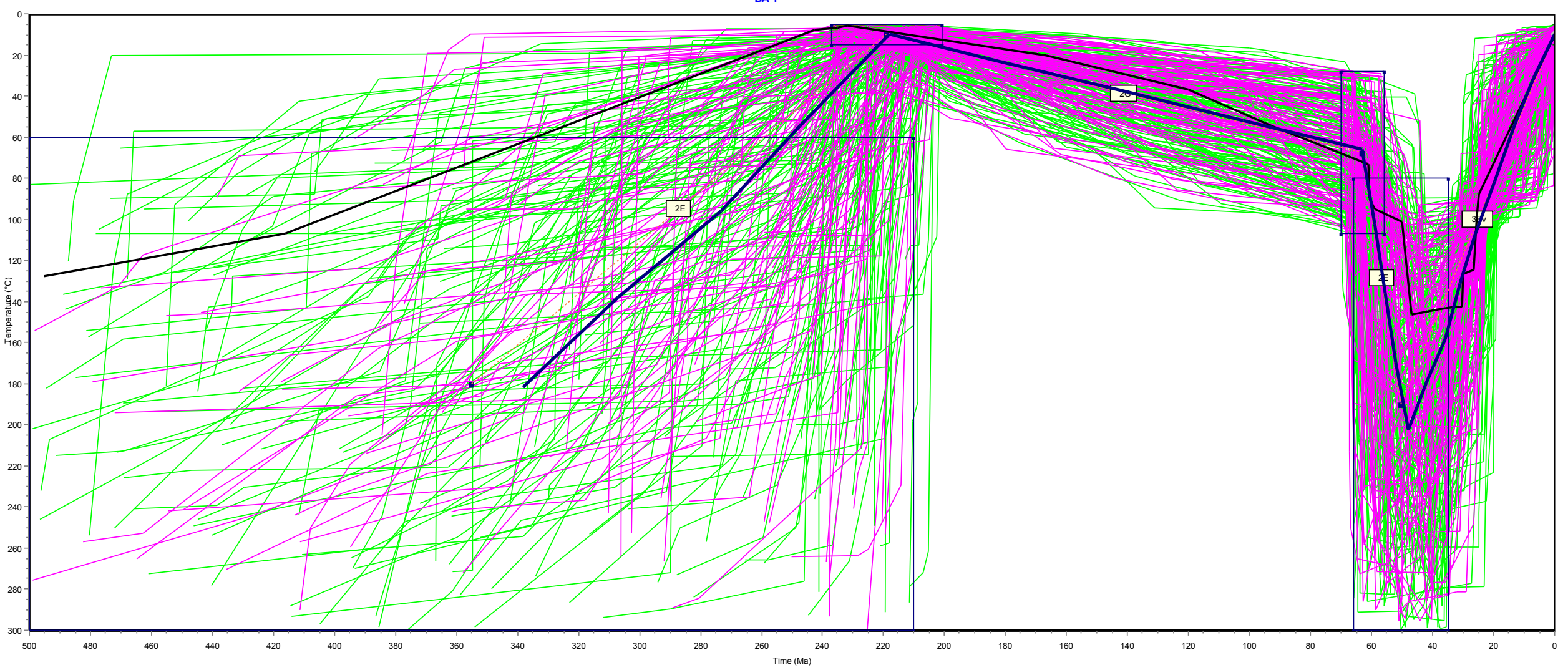

AFT

\begin{tabular}{llll} 
& & Measured age & Model age \\
\hline Simulated paths & 1193 & $25.1 \pm 3.2$ & 25.1 \\
Acceptable & 169 & & \\
Good & 100 & &
\end{tabular}


Figure S6: DER-2

DER-2

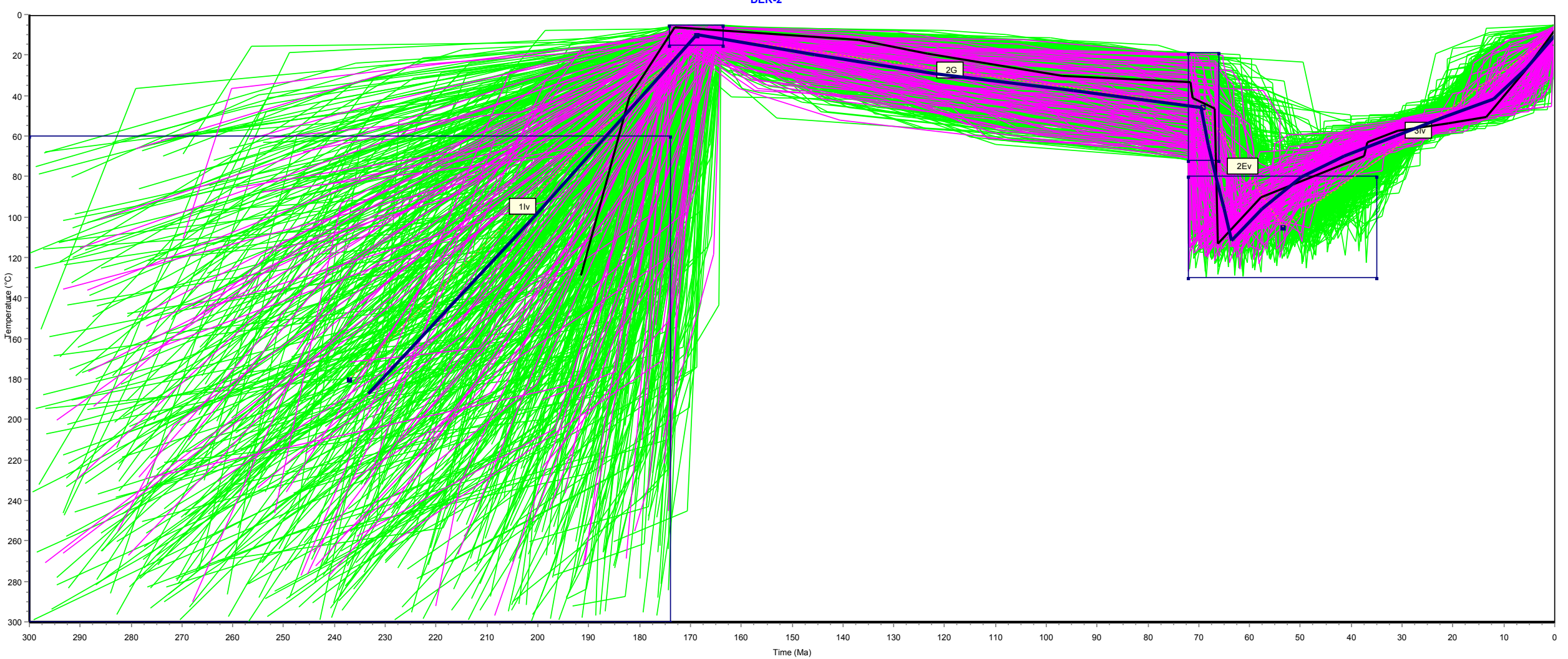

\begin{tabular}{|c|c|c|c|c|c|c|}
\hline & & $\begin{array}{l}\text { AHe } \\
\text { Measured age }\end{array}$ & Model age & AFT & Measured & Model \\
\hline Simulated paths & 183980 & $30.4 \pm 1.7$ & 30.2 & AFT age & $89.5 \pm 8.2$ & 89.5 \\
\hline Acceptable & 758 & $27.9 \pm 1.7$ & 26.4 & Track Length & $11.65 \pm 1.71$ & $10.98 \pm 3.54$ \\
\hline Good & 100 & $\begin{array}{l}24.3 \pm 1.4 \\
305+1.4\end{array}$ & $\begin{array}{l}24.3 \\
30\end{array}$ & GOF & 0.54 & \\
\hline
\end{tabular}




\section{Figure S7: SEY-3}

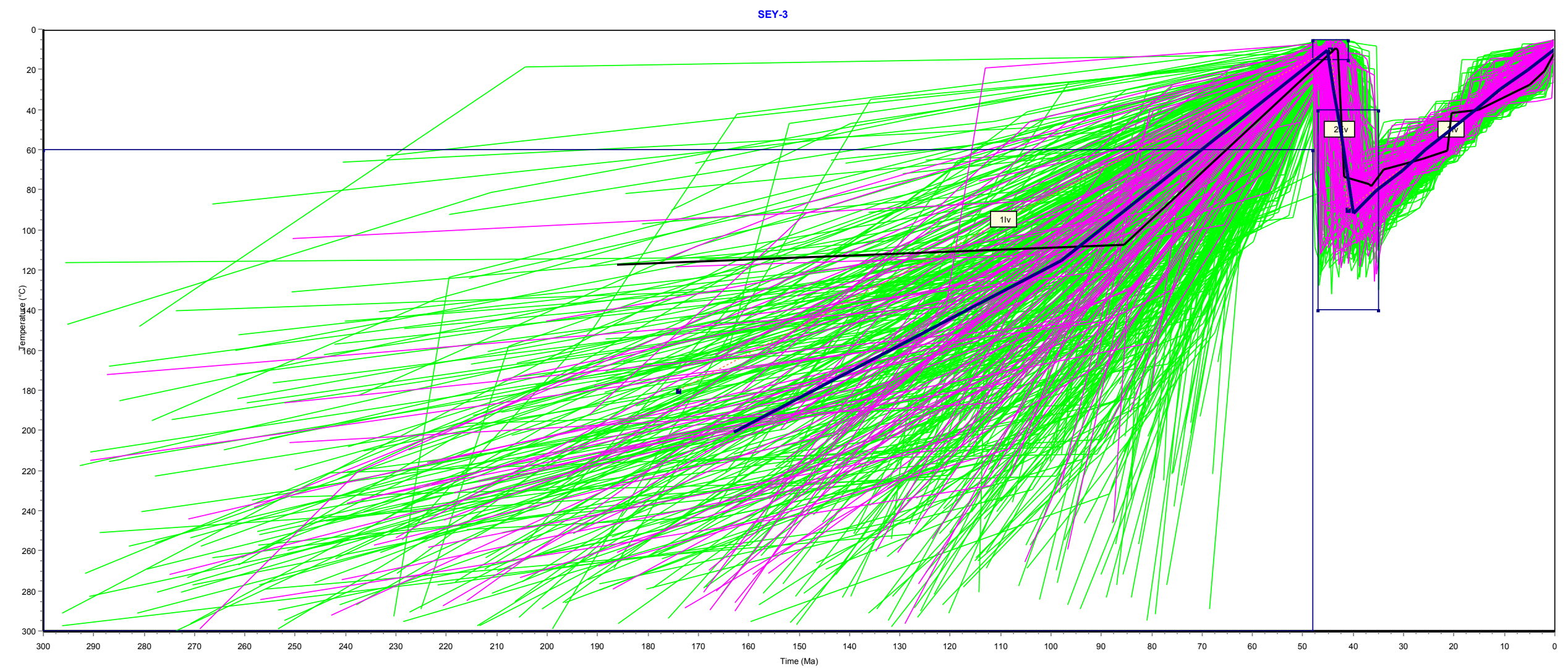

\begin{tabular}{llllll} 
& & AFT & & ZHe \\
& & Measured age & Model age & Measured age & Model age \\
\hline Simulated paths & 326042 & $44.9 \pm 7.6$ & 43.0 & $223.7 \pm 17.8$ & 236 \\
Acceptable & 1296 & & & $331.4 \pm 27.1$ & 329 \\
Good & 100 & & & $244.7 \pm 11.8$ & 244
\end{tabular}




\section{Figure S8: SEY-1}

SEY-1_ZR1

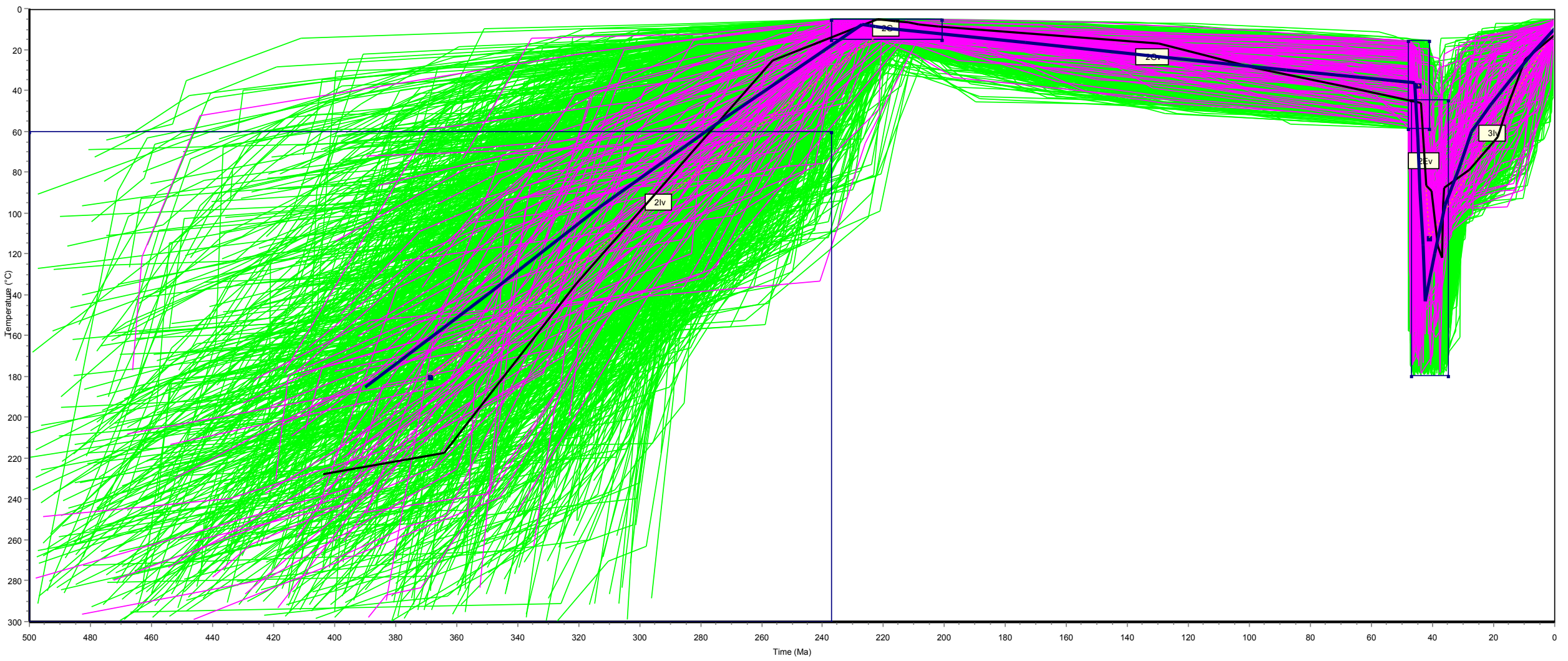

AFT ZHe

\begin{tabular}{llllll} 
& & AFT & & ZHe \\
& & Measured age & Model age & Measured age & Model age \\
\hline Simulated paths & 326042 & $44.9 \pm 7.6$ & 43.0 & $223.7 \pm 17.8$ & 236 \\
Acceptable & 1296 & & & $331.4 \pm 27.1$ & 329 \\
Good & 100 & & & $244.7 \pm 11.8$ & 244
\end{tabular}

